Case report

\title{
Conjunctival geographic ulcer and blepharitis in primary ocular herpes: a case report Rohina Swaroop
}

Address: 3501 Champion Lake Boulevard, \#1609, Shreveport, LA 71105, USA

Email: rohina123@gmail.com

Received: 3 June 2009 Accepted: 3 July 2009 Published: 29 July 2009

Cases Journal 2009, 2:814I doi: 10.4076/1757-|626-2-814I

This article is available from: http://casesjournal.com/casesjournal/article/view/8I4I

(c) 2009 Swaroop; licensee Cases Network Ltd.

This is an Open Access article distributed under the terms of the Creative Commons Attribution License (http://creativecommons.org/licenses/by/3.0), which permits unrestricted use, distribution, and reproduction in any medium, provided the original work is properly cited.

\begin{abstract}
Introduction: Conjunctival geographic ulcer is a rare manifestation of ocular herpes simplex. Geographic ulcers are formed when sloughing of the epithelium occurs in the areas between the dendrite and a broad area of epithelial involvement with irregular angulated borders is formed.

Case presentation: We report a case of primary ocular herpes with blepharitis and geographic ulceration of the conjunctiva in an 8-year-old male with no corneal lesion. To the best of our knowledge only 4 cases of conjunctival herpetic dendritic ulcerations and a single case of herpetic geographical ulcer have been reported in literature till date.

Conclusion: This is a rare clinical presentation of primary ocular herpes and ophthalmologists need to be aware and vigilant of this, as one of the myriad manifestations of primary ocular herpes, thereby providing appropriate therapy/treatment.
\end{abstract}

\section{Introduction}

HSV infection is the most common cause of blindness in developed countries. Primary infection with HSV-1 is silent in the majority of cases, with as few as $6 \%$ of cases being symptomatic .The lesions develop 10-12 days after exposure to the virus. Primary ocular herpes usually presents as blepharitis, blepharoconjunctivitis or keratitis. Conjunctival ulceration in the form of dendrites or geographic ulcers in ocular herpes is very rare.

In a study conducted in Rochester, Minnesota [1] over a period from 1950 to 1982 it was observed that Primary ocular herpes involved lid or conjunctiva in $54 \%$, superficial cornea in $63 \%$, deeper cornea in $6 \%$, and uveitis in $4 \%$. Darouger et al [2] in a study conducted in 108 patients of primary ocular herpes found $14(12.9 \%)$ patients to be in the age group 5-10 years of age.

Herpes simplex virus infections [3] involving the lid may present in one of two forms. The classic appearance involves an accumulation of small vesicles or pustules along the lid margin and/or periocular skin, itching may be associated symptom. These lesions have an inflamed, erythematous base. Within the first week of infection, the vesicles may ulcerate or harden into crusts. Once the crusts fall off the lesion is no longer infectious. Erosive-ulcerative form of HSV blepharitis has also been described. This presentation is characterized by erosions of the lid at the Gray line or ulcers along the lid margin, or a combination of both. Spread of the virus from eyelids and conjunctiva 


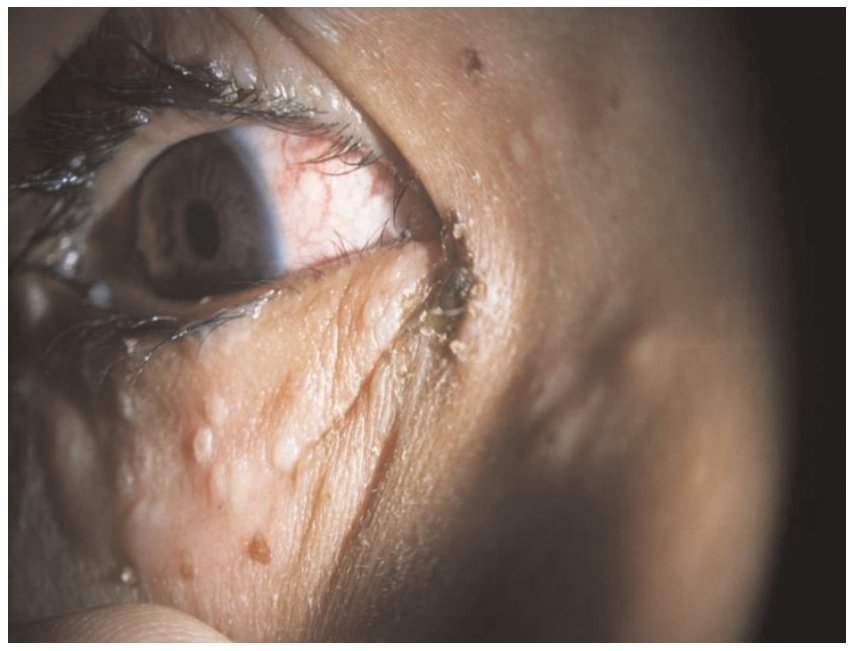

Figure I. Eruptions in the Right periorbital region. Small vesicles can be seen along the lid margin and periocular skin. Figure also shows crusting of the eyelids and conjunctival congestion.

to cornea is uncommon in children even without antiviral prophylaxis. A study conducted in Japan [4] even suggested that a biological difference may exist between HSV strains causing keratitis and conjunctivitis.

Conjunctival geographic ulcer is a rare manifestation of ocular herpes simplex. Geographic ulcers are formed when sloughing of the epithelium occurs in the areas between the dendrite and broad area of epithelial involvement with irregular angulated borders is formed. To the best of our knowledge 4 cases of conjunctival dendritic ulcerations $[5,6]$ and [7] a single case of herpetic geographical ulcer has

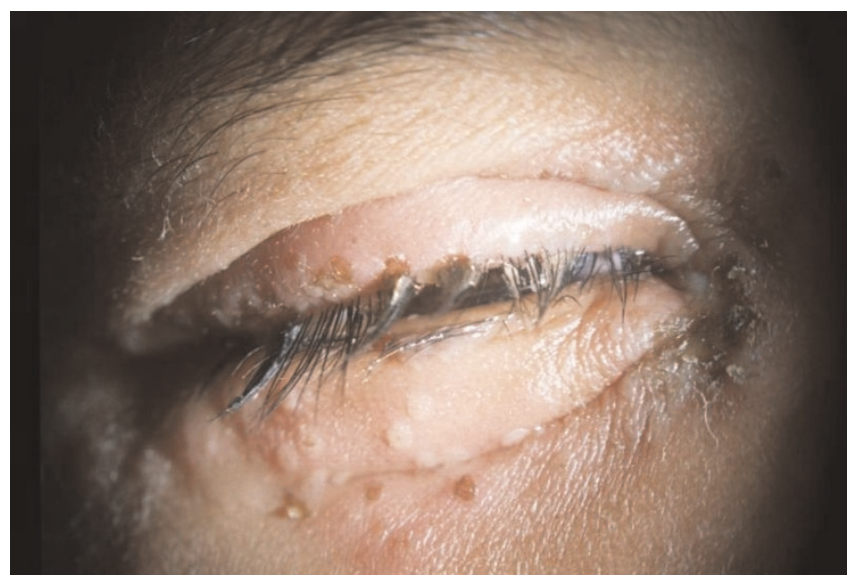

Figure 2. Herpetic blepharitis. Swelling and erythema of the eyelids along with erosions and ulcerations of the lid margins can be seen.

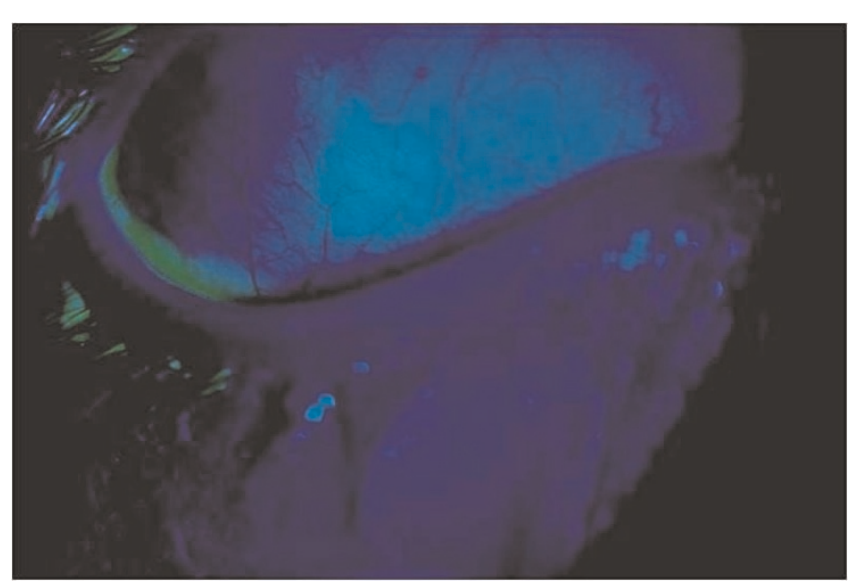

Figure 3. Fluorescein staining in the lower bulbar conjunctiva. Well defined geographic ulcer with irregular, angular, dendritiform margins can be seen on fluorescein staining of the lower bulbar conjunctiva.

been reported in literature till date. Only 1 of the reported cases of conjunctival dendrite had primary ocular herpes.

The reasons for herpetic lesions having a dendritiform and on progression of the disease, a geographic pattern are not known. Earlier it was postulated that the shape was due to herpes involving the corneal nerves. Another school of thought [8] was that it was due to "polygonal cells" in epithelium. The demonstration of dendrites on the conjunctiva refutes both these theories.

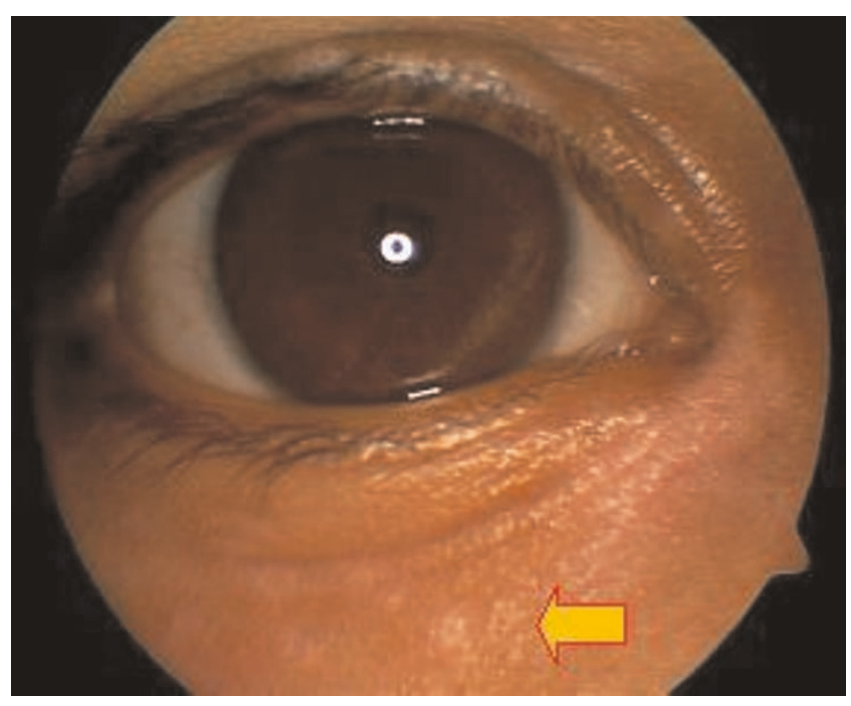

Figure 4. Healed blepharitis and periorbital skin lesions after 12 days of therapy. Only slight hypopigmentation at the site of the previous skin lesions can be observed after 12 days of therapy. 


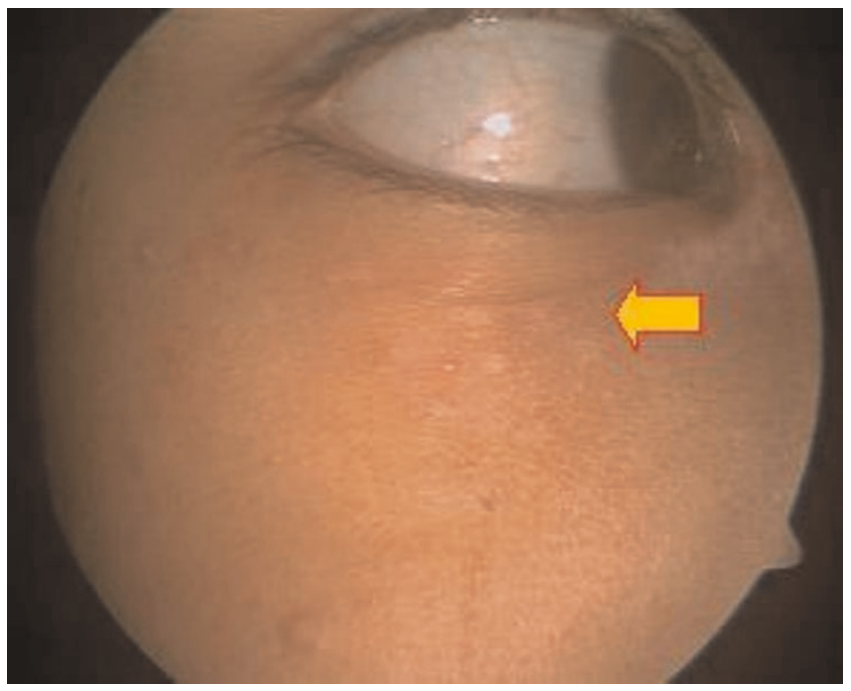

Figure 5. Healed blepharitis and periorbital skin lesions after 12 days of therapy. Only slight hypopigmentation at the site of the previous skin lesions can be observed after 12 days of therapy.

\section{Case presentation}

A 8-year-old Asian Indian male presented with complaints of pain, swelling and eruptions on right upper and lower lids for the last 7 days. No significant past medical/ surgical/ family/social history was elicited. No rhinitis/

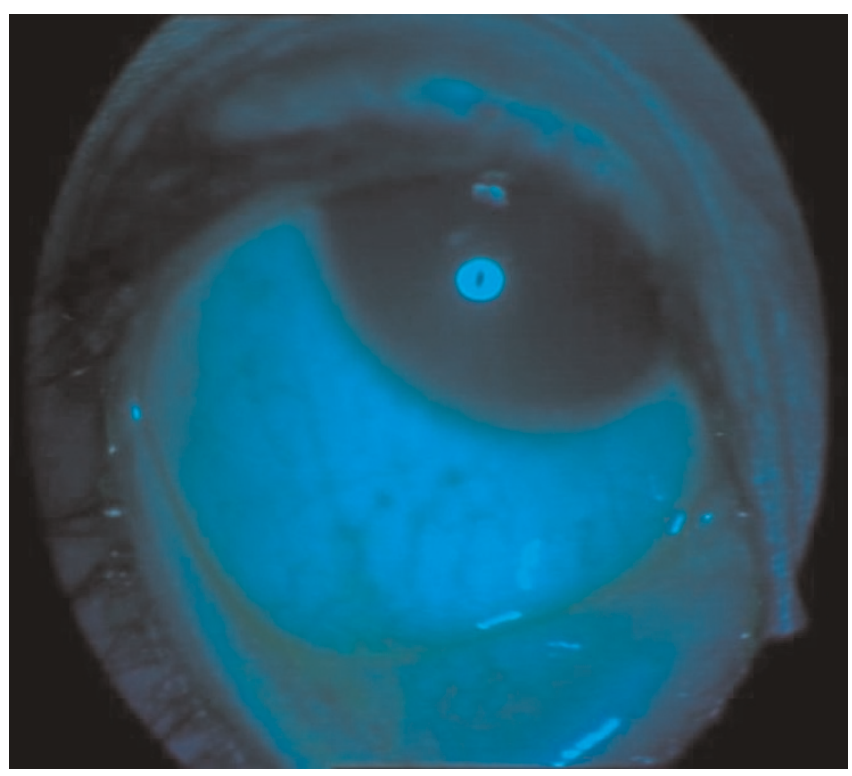

Figure 6. Healed conjunctival geographic ulcer after 12 days of therapy. No fluorescein staining in the lower bulbar conjunctiva after 12 days of therapy. Geographic ulcer has completely healed. pharyngitis/fever was present. No preauricular lymphadenopathy was present. Vision was $6 / 6$ in both eyes. Vesicular eruptions were present on right side of face near the periorbital region, and right upper and lower lids showed swelling and vesicular eruption. Corneal sensations were diminished in the right eye.

Slit lamp examination of the right eye with fluorescein staining showed well defined area of fluorescein staining in the lower bulbar conjunctiva resembling a geographic ulcer with irregular, angulated margins. Anterior segment and posterior segment examination were unremarkable. Patient was started on Acyclovir (3\%) ointment 5 times/ day local application on eye and skin and oral Acyclovir $200 \mathrm{mg} 5$ times/day. He was reviewed after 3 days and then after 12 days. Patient recovered completely after 12 days and medications were terminated.

\section{Conclusion}

This is a rare clinical presentation of primary ocular herpes and ophthalmologists need to be aware and vigilant of this, as one of the myriad manifestations of primary ocular herpes, thereby providing appropriate therapy/treatment.

\section{Abbreviations}

HSV, Herpes Simplex Virus; HSV-1, Herpes Simplex Virus type 1 .

\section{Consent}

Written informed consent was obtained from the patient's parents for publication of this case report and accompanying images. A copy of the written consent is available for review by the Editor-in-Chief of this journal.

\section{Competing interests}

The author has no financial or non-financial conflicts of interest pertinent to this case report.

\section{Acknowledgements}

Umang Mathur contributed towards the study by making substantial contributions to the conception, analysis and interpretation of data and was involved in revising the manuscript critically for important intellectual content.

\section{References}

I. Liesegang TJ, Melton LJ 3rd, Daly PJ, Ilstrup DM: Epidemiology of ocular herpes simplex. Incidence in Rochester, Minnesota, 1950 through 1982. Arch Ophthalmol 1989, 107:| I55-II59.

2. Darouger S, Wishart MS, Viswalingam ND: Epidemiological and clinical features of primary herpes simplex ocular infection. $\mathrm{Br} J$ Ophthalmol 1985, 69:2-6.

3. Handbook of Ocular Disease Management. [http://www. revoptom.com/HANDBOOK/SECT3 I a.HTM].

4. Uchio E, Takeuchi S, Itoh N, Matsuura N, Ohno S, Aoki K: Clinical and epidemiological features of acute follicular conjunctivitis with special reference to Herpes Simplex Virus type I. $\mathrm{Br} J$ Ophthalmol 2000, 84:968-972. 
5. Sridhar U, Bansal Y, Choudhury S, Gupta AK: Conjunctival dendrite in a case of Primary herpes simplex infection. $\mathrm{Br} J$ Opthalmol 2004, 88:590-59|.

6. Colin J, LeGrignou A, Baikoff G, Chastel F: Three cases of dendritic herpetic ulcerations of the conjunctiva. Am J Opthalmol 1980, 89:608-609.

7. Jain AK, Sukhija J, Chopra I, Sachdev N: Primary conjunctival herpetic geographic ulcer in an immunocompetent patient. Compr Ther 2007, 33:36-38.

8. Blaum JL: Morphogenesis of the dendritic figure in herpes simplex keratitis. Am J Ophthalmol 1972, 70:722-724.

\section{Do you have a case to share?}

Submit your case report today

- Rapid peer review

- Fast publication

- PubMed indexing

- Inclusion in Cases Database

Any patient, any case, can teach us something

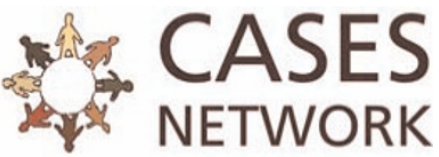

www.casesnetwork.com 